\title{
A study on the combination of singular cancellation and AIM
}

\author{
Ming-Xuan Zheng ${ }^{\dagger}$, Hui-Ling Zhao and Zhong-Hui Zhao \\ Department of Electronics and Information, \\ Northwest Polytechnical University, Xi'an, China \\ ${ }^{\dagger}$ E-mail: x408859786@126.com \\ www.nwpu.edu.cn
}

\begin{abstract}
Near-singularity and far-filed storage in impedance matrix were two difficulties in MoM. In this paper, some simple methods, based on the Duffy transformation, were effectively used in dealing with the near-singularity. For the far part, AIM(Adaptive Integration Method) was used to reduce not only the memory cost but also the time consuming in solving. The results show the accuracy of the cancellation method and the rapidity as well as low cost of AIM.
\end{abstract}

Keywords: AIM; Duffy Transformation; Near-singularity.

\section{Introduction}

The MoM [1](Method of Moments) was widely used in calculating radiation and scattering problems for the accurate analysis. However, the near-singular kernel involved in a pair of triangle elements, when they were overlap or closer. Moreover, the dense matrix for the impedance would dominate a lot of memory with the increasing unknowns, which will consume more time during iterative algorithm such as BiCGStab (Biconjugate Gradient Stable).

Some near-singularity cancellations were proposed in literature [4], the scheme of these methods was to eliminate the singular term by the Jacobian matrix derived from the transformation without any complex procedures. Additionally, based on the FFT(Fast Fourier Transform), AIM[5,6,8] could compress the elements in the far field of impedance matrix to save memory and accelerate matrix-vector multiplication in the iteration which was very suitable to deal with the large-size problems.

In this paper, the near-singularity will be focused firstly by some methods based on the Duffy transformation, which will be illustrated in section II. In order to deal with the large-size problem, AIM is introduced to compress the far field part and accelerate the matrix solving in section III. The following section will give some numerical validations of this combination algorithm. The conclusion will be summarized in the last section. 


\section{Singularity in the Near Field}

In MoM, when the field and source triangular patches are closer, especially when they are the same one, the green function will be infinite for its singular term as Eq.(1)

$$
\int_{S} \frac{d s}{R^{n}}
$$

where $R$ is the distance between field and distance, $n=1,2,3$ is for different cases and $S$ is the patch. It is difficult to receive a high accurate value through direct integration with the standard Guass quadrature.

The near-singularity-cancellation based on the Duffy transformation[2] will be used to deal with this problem by dividing the original triangular patches into three sub-triangles at first before constructing the local coordinate for the three sub-triangles.

The local coordinate, which is constructed on the subtriangle plane, $y$ and $x$ axis are parallel and orthogonal to the corresponding edge in the original triangle. Under the application of the Duffy transformation $u=x / y$, for one of the subtriangle $T$, the Eq.(1) will be transformed as:

$$
\int_{T} \frac{d x y}{\sqrt[n]{x^{2}+y^{2}+z^{2}}}=\int_{T} \frac{y d u d y}{\sqrt[n]{\alpha^{2} y^{2}+z^{2}}} \quad R=\sqrt{x^{2}+y^{2}+z^{2}}, \alpha^{2}=1+u^{2}
$$

However, the kernel pole will shift slightly after the Duffy transformation, which means the error will be converged more slowly with using the standard Guassian quadrature.

A little modification starts from Duffy transformation as Eq.(3)

$$
\frac{d u}{\sqrt[n]{1+u^{2}}} \cdot \frac{y d y}{\sqrt[n]{y^{2}+z^{2} /\left(1+u^{2}\right)}}
$$

Then the space $(x, y)$ is transformed into the space $(w, v)$ under the limitation of integration given in Eq.(4), where the Jaccobi Matrix will cancel the singularity:

$$
\begin{aligned}
& w=w(x / y), v=v(y, w) \\
& \left\{\begin{array} { l } 
{ w _ { l } = w ( x _ { 2 } / y _ { 2 } ) } \\
{ w _ { u } = w ( x _ { 1 } / y _ { 1 } ) }
\end{array} \left\{\begin{array}{l}
v_{l}=v(0, w) \\
v_{u}=v(h, w)
\end{array}\right.\right.
\end{aligned}
$$

where $l$ and $u$ stand for the low and up limitation. For different cases $(n=1,2,3)$, the detail formulations, ADRNC(Augmented-Duffy- $R^{n}$-Constant scheme) and ADRNL(Augmented-Duffy- $R^{n}$-Linear scheme), can be found in Ref.[3]. 


\section{AIM}

In order to solve the large-size problem under the low memory requirement and fast speed, the matrix-vector multiplication of AIM, compressing the far field impedance in a multiplication of several spares matrix, is accelerated during the iteration by FFT and inverse FFT for EFIE(Electric Field Integration Equation) as Eq.(5):

$$
\begin{aligned}
{[\mathrm{Z}][I] } & =\left[Z^{\text {near }}\right][I]+[\mathrm{Z}]^{\text {far }}[I] \\
& =\left[Z^{\text {near }}\right][I]+j \omega \mu\left(\sum_{r=x, y, z}\left[\Lambda_{r}\right] \operatorname{IFFT}\left\{\tilde{\mathbf{G}} \cdot F F T\left[\left[\Lambda_{r}\right]^{T}[I]\right]\right\}\right. \\
& \left.-\frac{1}{k^{2}}\left[\Lambda_{d}\right] \operatorname{IFFT}\left\{\tilde{\mathbf{G}} \cdot F F T\left[\left[\Lambda_{d}\right]^{T}[I]\right]\right\}\right)
\end{aligned}
$$

where $\omega$ is the angle frequency, $\mu$ and $k$ are the permeability and the wave number, respectively. $\left[Z^{\text {near }}\right]$ is the near field elements after the near correction, which is kept in a large spares matrix with a little memory. All the matrices of $[\Lambda]$ are the expansion coefficients gained by multipole expansion for every basic function, which are also saved in several spares matrices. With the property of multilevel Teoplitz[7], $\widetilde{\mathbf{G}}$ is the vector of Green function [G] after FFT. In fact, $\left[Z^{f a r}\right]$, represented by the multiplication of $[\Lambda]$ and $[G]$, is an good approximation for that of MoM when the distance between two patches is just larger than $0.3 \lambda$. It means a huge number of elements in the dense impedance of MoM will be saved in a compressing form.

Compared with MoM, the memory requirement and the complexity of AIM decline from $O\left(N^{2}\right)$ to $O(N)$ and from $O\left(N^{2}\right)$ to $O(N \log N)$ for the 2D problem respectively, which provide the high speed and low cost memory for the largesize electromagnetic problem.

\section{Numerical Results}

Firstly, to validate the accuracy of the near-singularity method in different $\operatorname{kernel}(n=1,2,3)$, the test triangle places on the xoy plane with the two edges valued by 1 along the $x$ and $y$ direction, respectively. The observation points is over the center of the test triangle at height $z$. 


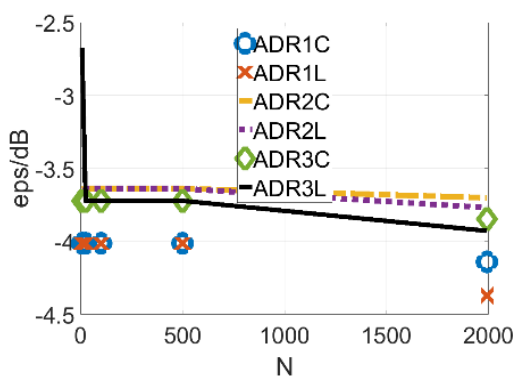

(a)

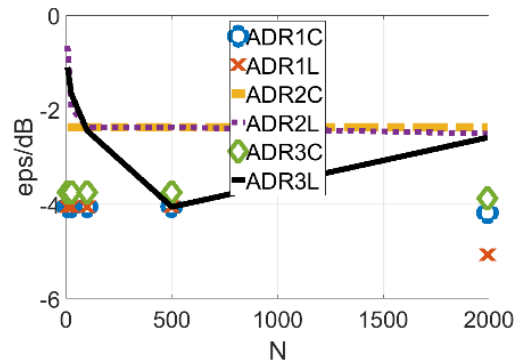

(b)

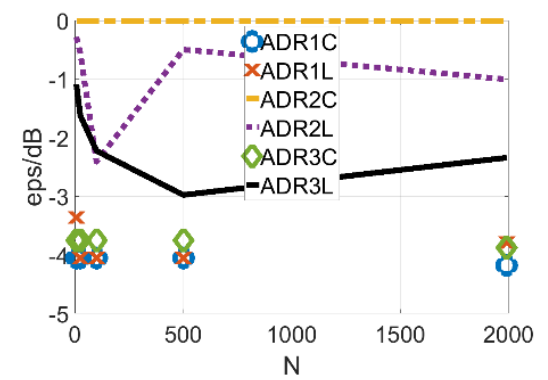

(c)

Fig. 1. The accuracy of the six cancellation methods in different distance between observation and the triangle $(\mathrm{a}) z=0.1$ (b) $z=0.001$ (c) $z=10^{-9}$. eps $(\mathrm{dB})$ is the relative error compared with standard Guass quadrature with more than 5000 points.

The accuracy versus the quadrature points of six methods for the three different distance between the observation and triangle at $z=0.1,0.001,10^{-9}$ are shown in Fig.1. All the methods converge quickly after the number of quadrature points larger than 25 when $z$ equals to 0.1 . For a closer distance at $z=0.001$, ADRC1 and ADRL1 are still the reliable methods for $n=1,2,3$ with less quadrature points while the curve of ADRL3 shows a rising after 500 points. It is noted that the perfect results will be achieved with fewer quadrature points when the distance is larger. However, Fig.1(c) suggests that the ADR3C and ADR3L are less accurate when the observation is almost at the triangle, which is more significant for ADR2C.

Next, for a pair of two PEC squares with the edges of $5 \lambda$ and $10 \lambda$, a comparison on memory requirement and cost time between AIM and MoM are listed in Table.1. 
Table 1. The comparison of AIM and MoM on memory and cost time for different square edges on $5 \lambda$ and $10 \lambda$

\begin{tabular}{lll}
\hline $5 \lambda / 10 \lambda$ & AIM & MoM \\
\hline Unknowns & $7364 / 30013$ & $7364 / 30013$ \\
Memory requirement(MB) & $6.65 / 17.85$ & $413 / 6872.41^{*}$ \\
Solving time(s) & $247.51 / 2756.02$ & $693.72 / * *$ \\
Spares rate of impedance matrix $(\%)$ & $98.98 \% / 98.74 \%$ & 0 \\
\hline$*$ estimated memory & & \\
$* *$ Cannot give the solving time & &
\end{tabular}

In Table.1, high spares rate $(98.98 \%$ and $98.74 \%)$ indicate that the memory requirement of AIM is lower than that of MoM by compressing most elements of impedance matrix. When the unknowns increase, it is estimated more than 6.7GB memory will be taken by MoM while only $17.85 \mathrm{MB}$ is needed by AIM. Moreover, it is suggested that the solving time will be shorter on AIM by the help of FFT and inverse FFT.

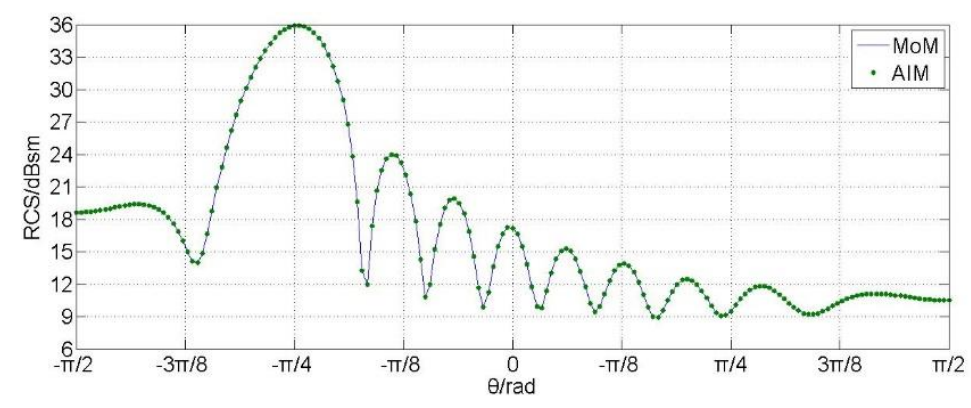

Fig. 2. The RCS of $5 \lambda \times 5 \lambda$ PEC square for $\varphi=0$ plane

Fig. 2 shows the RCS of the square in $x o z$ plane, whose edges' length are $5 \lambda$ with the incident plane wave in the direction of $\theta=\pi / 4$ and $\varphi=0$. It is not surprised that the highest reflection is at the $\theta=-\pi / 4$ and the calculation of AIM agrees with that of MoM.

\section{Conclusion}

A combination of near-singularity cancellation methods and AIM for near and far parts of impedance is illustrated in this paper. In the near field, when the observation point close to the triangle, the methods for $n=1$ are much more accurate with few Guass quadrature points, while the ADR3C and ADR3L are worse. Compared with that of MoM, the memory requirement and time consumption of AIM decrease dramatically and the performance is rather well. 


\section{Acknowledgement}

This work is supported by 20142053025 of the aeronautical science foundation of China.

\section{References}

1. R. F. Harrington, Field Computation by Moment Methods(New York: MacMlillan, 1968).

2. M. G. Duffy. Quadrature over a pyramid or cube of integrands with a singularity at a vertex. SIAM J. Numer. Anal. 19.1260-1262(1982).

3. Botha, M. M. A Family of Augmented Duffy Transformations for NearSingularity Cancellation Quadrature. Antennas and Propagation, IEEE Transactions on. 61,3123-3134(2013).

4. Botha, M. M. and Strydom, W. J. Integrating the dynamic MoM kernel with near-singularity cancellation quadrature schemes. In AFRICON, pp. 13(2013).

5. Bleszynski, E., Bleszynski, M. and Jaroszewicz, T. AIM: Adaptive integral method for solving large-scale electromagnetic scattering and radiation problems. Radio Sci. 31,1225-1251 (1996).

6. Wei-Bin, E., Le-Wei, L., Qun, W. and Mook-Seng, L. AIM solution to electromagnetic scattering using parametric geometry. Antennas and Wireless Propagation Letters, IEEE. 4,107-111(2005).

7. Barrowes, B. E., Teixeira, F. L. and Kong, J. A. Fast algorithm for matrixvector multiply of asymmetric multilevel block-toeplitz atrices. Antennas and Propagation Society International Symposium, IEEE. 4,630-633(2001).

8. Kai, Y. and Yilmaz, A. E. An FFT-Accelerated Integral-Equation Solver for Analyzing Scattering in Rectangular Cavities. Microwave Theory and Techniques, IEEE Transactions on. 62,1930-1942 (2014). 